\title{
Gamma Irradiation-Assisted Synthesis of Cellulose Nanocrystal-Reinforced Gelatin Hydrogels
}

\author{
Wan Hafizi Wan Ishak ${ }^{1}$, Ishak Ahmad ${ }^{1, *}$, Suria Ramli ${ }^{1}$ and Mohd Cairul Iqbal Mohd Amin ${ }^{2}$ \\ 1 Polymer Research Centre (PORCE), School of Chemical Sciences and Food Technology, \\ Faculty of Science and Technology, Universiti Kebangsaan Malaysia, Bangi Selangor 43600, Malaysia; \\ hafizi.wan29@gmail.com (W.H.W.I.); su_ramli@ukm.edu.my (S.R.) \\ 2 Faculty of Pharmacy, University Kebangsaan Malaysia, Jalan Raja Muda Abdul Aziz, Kuala Lumpur 50300, \\ Malaysia; mciamin@ukm.edu.my \\ * Correspondence: gading@ukm.edu.my; Tel.: +60-3-8921-5420
}

Received: 28 August 2018; Accepted: 18 September 2018; Published: 21 September 2018

check for updates

\begin{abstract}
Herein, we describe the use of gamma irradiation to prepare hydrogels comprising $\alpha$-cellulose and cellulose nanocrystal (CNC)-reinforced gelatin in the absence of crosslinking agents. In this study, cellulose was extracted from rice husks by an alkali and bleaching treatment followed by acid hydrolysis to produce CNC. A semi-interpenetrating network (semi-IPN) of hydrogels was developed by the miscibility between gelatin and cellulosic materials. Compared to those prepared from $\alpha$-cellulose, hydrogels prepared by dispersion of CNCs exhibited remarkably enhanced stiffness and swelling properties, which was ascribed to the uniform distribution of CNCs and their increased crystallinity. Improved pore structure, arrangement, and rigidity of CNC-reinforced gelatin hydrogels, which induced the swelling mechanism resulting in higher and faster water uptake was observed with a scanning electron microscope (SEM), compared to cellulose-reinforced gelatin hydrogels. Moreover, in vitro drug profiling demonstrated that $\mathrm{CNC} /$ gelatin hydrogels exhibit good drug loading/release behavior and are thus suitable for use in drug-delivery applications.
\end{abstract}

Keywords: hydrogels; cellulose nanocrystal; gelatin; radiation

\section{Introduction}

Gelatin is a nontoxic, biodegradable, cheap, and nonimmunogenic biopolymer with promising pharmaceutical and medicinal applications. Being a derivative of (naturally occurring) collagen, gelatin exhibits superb biocompatibility and thus attracts increased attention as a material suitable for potential biomedical applications, as exemplified by the recently described gelatin-based hydrogels [1].

However, gelatin suffers from certain drawbacks, e.g., it cannot withstand the normal body temperature of $37^{\circ} \mathrm{C}$ due to undergoing a sol-gel transition under these conditions, which limits its biological and medical applications [2]. Moreover, gelatin exhibits low in vivo mechanical strength and elasticity as a result of swelling, which, nonetheless, can be mitigated by crosslinking to improve both thermal and mechanical stabilities [3].

Among the variety of available crosslinking methods (physical, chemical, and radiation-nduced) [4], chemical crosslinking, based on the introduction of chemical additives such as glutaraldehyde and formaldehyde, is the one most commonly used. However, the presence of such chemical residues in a gelatin structure results in cytotoxicity [5], which makes chemically crosslinked gelatin unsuitable for biomedical and pharmaceutical applications.

In view of the above, radiation-induced crosslinking appears to be the best possible method due to not involving the use of any chemical reagents or additives. Specifically, gamma radiation is well known to induce gelatin crosslinking [5] and afford a three-dimensional network by forming chemical 
bonds between molecular backbones [6]. Advantages of radiation-induced polymer degradation include its ability to promote reproducible and quantitative changes without the introduction of chemical reagents and concomitantly occurring product sterilization [7]. In addition to crosslinking, the incorporation of natural fibers (such as cellulose) into gelatin helps to improve the mechanical and thermal properties of the corresponding hydrogels. Generally, radicals are formed from chain scission of the polymer chain in high-energy crosslinking, especially when irradiating dried gelatin or cellulose in the presence of oxygen [8]. The degradation of both polymers credited by the chain scission does not occur without an oxygen environment during irradiation, particularly when irradiating in solution. Prior to crosslinking, the restriction of oxygen and the presence of water also result in the termination of radicals, reflecting both gelatin and cellulosic material, respectively [8,9]. Lepore et al. [10] reported that the amorphous region of cellulose easily trapped water molecules, while water was present in the crystalline region. In this study, the irradiation process was done with the restriction of oxygen and the presence of water to terminate radicals and promote the crosslinking process. Similar procedures have been used for different polymers to produce hydrogels $[5,8]$.

Cellulose is one of the best known naturally occurring polysaccharides, being the most earth-abundant polymer and exhibiting advantages of renewability, biodegradability, and nontoxicity [11]. The unique semicrystalline nature of cellulose allows its fibers to be reduced in size and form cellulose nanocrystals (CNCs) upon partial acid hydrolysis. Under controlled conditions, the acid-catalyzed cleavage of cellulose fibers occurs at amorphous regions, leaving highly crystalline segments intact, which results in the production of rodlike nanometer-scale particles (also known as whiskers). Currently, increased attention has been directed at the preparation and applications of nanocellulose as a polymer-reinforcing material [12].

Over the past decade, the use of biopolymer hydrogels produced from synthetic or natural polymers for biomedical and food applications has experienced tremendous popularity increase and has become a hotspot of interest for many research groups worldwide, with notable examples including wound dressing [13], drug delivery [14], and tissue-engineering scaffolds [15]. Herein, we developed a novel green technology of preparing CNC- and original cellulose fiber-reinforced gelatin hydrogels utilizing gamma radiation to determine the effect of cellulosic particle size on hydrogel performance, hypothesizing that the presence of variable-size cellulosic fibers (i.e., micro- and nanocellulose) alters the water-absorption capacity and other properties of these hydrogels. Moreover, incorporation of CNCs was expected to increase the rigidity of the soft-hydrogel network and improve its water-uptake ability, making the thus-obtained crosslinked hydrogels useful for future biomedical applications.

\section{Materials and Methods}

\subsection{Materials}

Pharmaceutical-grade gelatin was purchased from Halagel (M) Sdn. Bhd. (Kuala Lumpur, Malaysia); sulfuric acid and rice-husk fibers were obtained from Sigma-Aldrich (Kuala Lumpur, Malaysia) and Bernas Sdn. Bhd (Shah Alam, Selangor, Malaysia), respectively.

\subsection{Preparation of Cellulose Fibers and Cellulose Nanocrystals (CNCs)}

$\mathrm{CNCs}$ were extracted from rice husks and characterized as described elsewhere [16]. Raw rice-husk fiber was ground to obtain rice husk powder (125-150 $\pm 5.5 \mu \mathrm{m})$, which was subsequently stirred in $4 \mathrm{wt} \%$ aqueous $\mathrm{NaOH}$ for $2 \mathrm{~h}$ at $80^{\circ} \mathrm{C}$ using a mechanical stirrer. The separated fibers were filtered and washed with distilled water to remove alkali-soluble components, and residual lignin in the alkali-treated powder was removed by bleaching, which was performed by $4 \mathrm{~h}$ stirring in acetate buffer $/ 2.7 \mathrm{wt} \% \mathrm{NaClO}_{2} /$ distilled water at $80{ }^{\circ} \mathrm{C}$. After sixfold repetition of the bleaching treatment, the remaining cellulose fibers were recovered as described above. CNCs were prepared by $30 \mathrm{~min}$ acid hydrolysis of cellulose fibers in $65 \mathrm{wt} \% \mathrm{H}_{2} \mathrm{SO}_{4}$ at $50{ }^{\circ} \mathrm{C}$ under continuous stirring. The resulting suspension was diluted with cold distilled water to stop the reaction and centrifuged for $10 \mathrm{~min}$ at 
$10,000 \times g \mathrm{rpm}$. The obtained reaction mixture was dialyzed against distilled water until constant $\mathrm{pH}$, and the thus-produced suspensions were freeze-dried to produce $\mathrm{CNC}$ powder.

\subsection{Hydrogel Fabrication}

Three hydrogels were prepared, namely, gelatin hydrogel (100\% gelatin), hydrogel A (cellulose/gelatin), and hydrogel B (CNCs/gelatin). Cellulose and CNCs (4\%) were separately mixed with distilled water, and the resulting mixtures were homogenized (IKA T-25 homogenizer, IKA-Werke $\mathrm{GmbH}$, Staufen im Breisgau, Germany) for $5 \mathrm{~min}$ at $8000 \mathrm{rpm}$ and ultrasonicated (Bransonic CPXH, Branson Ultrasonic, Danbury, CT, USA) for $10 \mathrm{~min}$ to obtain homogeneous dispersions. Subsequently, these dispersions were treated with gelatin (96\%) and stirred with a magnetic stirrer at $55^{\circ} \mathrm{C}$ until homogeneity. The thus-prepared mixtures were exposed to gamma radiation (Gamma Cell 220 Excel, MDS Nordion, Ottawa, ON, Canada) at a dose of $30 \mathrm{kGy}$ (irradiation dose was carried out in accordance with the Food and Drug Administration [17]). Samples were irradiated at $25{ }^{\circ} \mathrm{C}$ inside sealed, airtight bags to prevent the presence of oxygen during irradiation.

\subsection{Morphological Studies}

CNC morphology was investigated by transmission electron microscopy (TEM) imaging (Philips CM 30, North Billerica, MA, USA). Contrast enhancement was achieved by staining nanocrystals with $2 \mathrm{wt} \%$ aqueous uranyl acetate solution for $1 \mathrm{~min}$. Cellulose was placed on an $\mathrm{Al} \mathrm{stub}$ and incubated in an oven at $60^{\circ} \mathrm{C}$, and the surface morphology of the thus-obtained samples was observed by scanning electron microscopy (SEM; Philips XL 30, North Billerica, MA, USA). Hydrogels were shock-frozen with liquid nitrogen and freeze-dried, with hydrogel morphology subsequently observed using variable-pressure SEM (VP Leo 1450; 500×, 15 kV, Oberkochen, Germany).

\subsection{X-ray Diffraction (XRD) Analysis}

$\mathrm{CNC}$ and cellulose fibers in the form of milled powders were subjected to room-temperature XRD analysis (D8-Advance Bruker AXS GmbH, Oestliche, Rheinbrueckenstr, Karlsruhe, Germany; $\mathrm{Cu} K_{\alpha}$ radiation $\left.(\lambda=0.1539 \mathrm{~nm}) ; 2 \theta=5-80^{\circ}\right)$, and their crystallinity indices (CrI) were determined by Diffrac. Suite EVA 1.4 software

\subsection{Fourier Transform Infrared (FTIR) Spectroscopy}

FTIR spectra were recorded in attenuated total reflectance (ATR) mode at room temperature in the range of $4000-500 \mathrm{~cm}^{-1}$ on a model 2000 Perkin Elmer instrument (Hopkinton, MA, USA) equipped with a diamond ATR crystal. For characterization, samples were cut into $1 \mathrm{~cm} \times 1 \mathrm{~cm}$ specimens ( $2 \mathrm{~mm}$ thick) and placed on the ATR plate.

\subsection{Rheological Characterization}

Hydrogels were prepared by immersion into distilled water at room temperature 2 days before analysis, and their rheological properties were characterized using a rheometer (Anton Paar Physica MRC 301, Anton Paar GmbH, Graz, Austria) with a $14 \mathrm{~mm}$ diameter flat plate. Storage moduli $\left(G^{\prime}\right)$ were determined at constant temperature $\left(25^{\circ} \mathrm{C}\right)$ and $0.05 \%$ shear strain for frequencies of $0.1-10 \mathrm{~Hz}$.

\subsection{Swelling Degrees}

The swelling degrees of hydrogels were determined by evaluating their ability to swell in distilled water at room temperature $\left(25^{\circ} \mathrm{C}\right)$ for $48 \mathrm{~h}$. The samples were dried before being dissolved in distilled water.

$$
\text { Swelling }(\%)=\frac{G_{\mathrm{s}}-G_{\mathrm{d}}}{G_{\mathrm{d}}} \times 100 \text {, }
$$


where $G_{\mathrm{s}}$ is the sample weight after $48 \mathrm{~h}$ swelling in distilled water, and $G_{\mathrm{d}}$ is the sample weight before immersion.

\subsection{In Vitro Drug Profiling}

A separation-diffusion method [18] was used to entrap riboflavin (model drug) into hydrogels. To determine drug-loading efficiency, disc-shaped hydrogel samples were immersed into $10 \mathrm{mg} / \mathrm{mL}$ of riboflavin solution for $24 \mathrm{~h}$, washed with $10 \mathrm{~mL}$ of distilled water, and dried in an oven for $24 \mathrm{~h}$ at $40{ }^{\circ} \mathrm{C}$. The drug concentration in the residual solution was analyzed by UV-vis spectrophotometry (UV-1800, Shimadzu, Kyoto, Japan) at a wavelength of $445 \mathrm{~nm}$, and drug-loading efficiencies (DL\%) were calculated as

$$
\mathrm{DL}(\%)=\frac{W_{\mathrm{dg}}}{W_{\mathrm{g}}} \times 100,
$$

where $W_{\mathrm{dg}}$ is the amount of drug loaded into the hydrogel, and $W_{\mathrm{g}}$ is the initial drug amount.

Drug-release profiles were obtained by immersing drug-loaded hydrogels into $50 \mathrm{~mL}$ of simulated intestinal fluid (SIF; $\mathrm{pH} 7$ ) at $37^{\circ} \mathrm{C}$ for $24 \mathrm{~h}$ under continuous stirring. At each predetermined time interval, $2 \mathrm{~mL}$ of this solution was removed for analysis. The drug solution was placed in a locked tube before it was determined by UV-vis spectrophotometry at the maximum absorbance wavelength of riboflavin $(445 \mathrm{~nm}$ ). For every removed aliquot, $2 \mathrm{~mL}$ of fresh SIF was added to maintain the volume of the remaining solution.

\section{Results}

\subsection{Morphological Analysis}

Sample morphology was analyzed by TEM as previously described for nanomaterial [19]. Figure 1 shows that upon acid hydrolysis, the diameter of individual cellulose fibrils was reduced from $4-8 \pm 1.32 \mu \mathrm{m}$ (pristine fibers) to $5-15 \pm 2.77 \mathrm{~nm}$ (CNCs), as previously reported [20]. The formation of crystalline rodlike or needlelike particles after partial acid hydrolysis was ascribed to the cleavage of the amorphous region of cellulosic microfibrils [11].

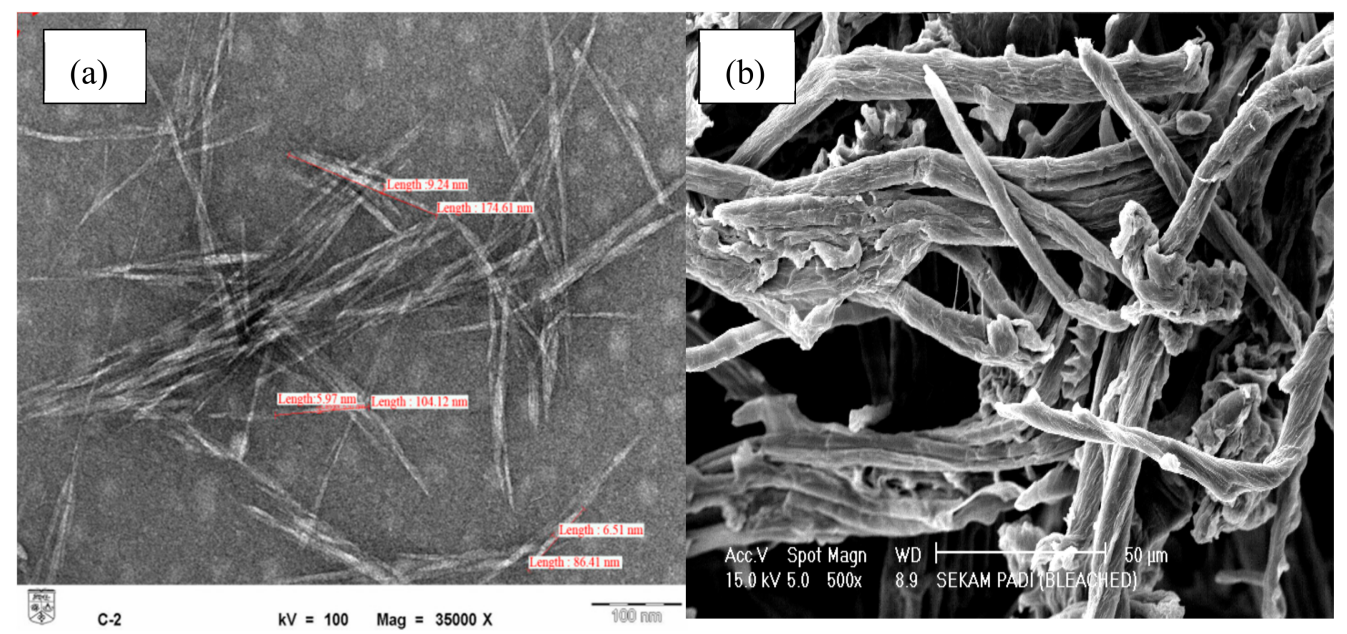

Figure 1. (a) Transmission electron microscopy (TEM) image of cellulose nanocrystals (CNCs) and (b) scanning electron microscopy (SEM) image of microcellulose fibers.

Each individual fibril of cellulose size was reduced from micrometer (Figure 1b) into nanometer (Figure 1a) range after acid hydrolysis treatment. The efficiency of acid hydrolysis treatment was demonstrated by the TEM micrograph (Figure 1a) where a needlelike structure of individual fibers with nanometer size was obtained. Similar results were also reported by Johar et al. [20]. A crystalline rodlike or needlelike particle remained after partial acid hydrolysis due to the cleavage of the amorphous 
region of cellulosic microfibrils [11]. The obtained CNCs had an aspect ratio of $10-25$, with $80 \%$ of them having an aspect ratio of 10-20. Thus, the produced CNCs could be well-dispersed in the polymer matrix due to their nanoscale size and thus facilitate the formation of stable-dimension pores in hydrogels to increase their water-absorption capacity [21]. Moreover, the nanoscale size of CNCs was also expected to provide superior surface area for the reaction to occur.

\subsection{XRD Analysis}

Chemical treatment of semicrystalline cellulose (e.g., strong acid-catalyzed hydrolysis) is well-known to influence fiber crystallinity due to inducing the formation of nanocrystals by removal of amorphous parts while keeping the crystalline region intact [22,23]. Herein, differences between the crystallinity of cellulose and CNCs were determined by XRD analysis (Figure 2).

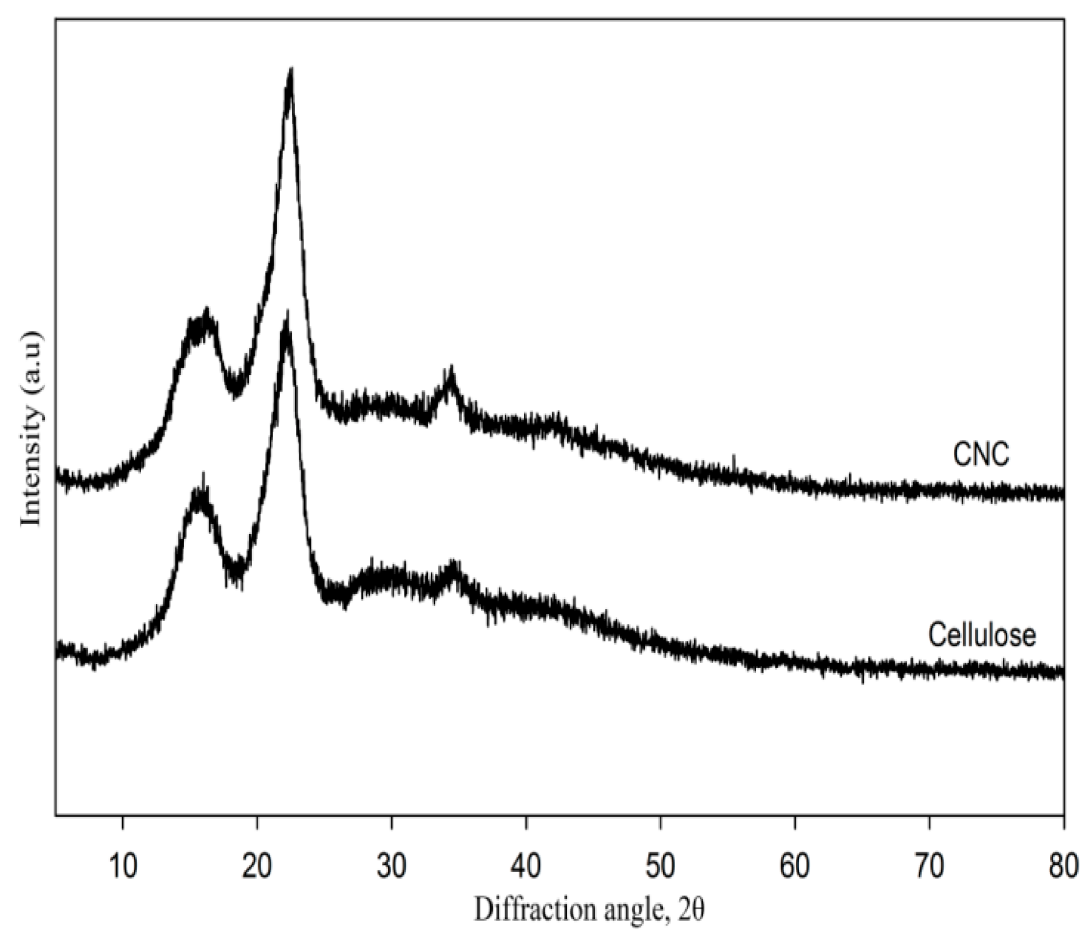

Figure 2. X-ray diffraction patterns of cellulose and CNCs.

The three well-defined crystalline peaks observed around $2 \theta=16^{\circ}, 22^{\circ}$, and $35^{\circ}$ were typical of cellulose I, being more intense and sharp for CNCs than for cellulose fiber and thus indicating the success of acid hydrolysis treatment [20]. Thus, hybridization of gelatin-based hydrogels with CNCs was expected to result in stiffness and rigidity, exceeding those obtained in the case of cellulose, i.e., the use of CNCs as reinforcing materials was assumed to increase the mechanical stability of the hydrogel itself. The crystallinity index (CrI) values for all peaks are summarized in Table 1.

Table 1. CrI values for cellulose and CNC XRD peaks.

\begin{tabular}{cccc}
\hline \multirow{2}{*}{ Fiber } & \multicolumn{3}{c}{ CrI (\%) at 20 } \\
\cline { 2 - 4 } & $\mathbf{1 6}^{\circ}$ & $\mathbf{2 2}^{\circ}$ & $\mathbf{3 5}^{\circ}$ \\
\hline Cellulose & 42 & 72 & 22 \\
CNCs & 54 & 74 & 37 \\
\hline
\end{tabular}




\subsection{FTIR Analysis}

FTIR characterization was carried out to confirm the crosslinking of gelatin and determine the cellulose reinforcement-induced change of functional groups therein (Figure 3). The CNC spectrum showed absorption peaks at 3335, 1060-1050, and $898 \mathrm{~cm}^{-1}$ corresponding to $\mathrm{O}-\mathrm{H}, \mathrm{C}-\mathrm{H}$, and $\mathrm{C}-\mathrm{O}$ stretches, respectively, in agreement with the results of a previous study [24]. No significant differences were observed between the spectra of original cellulose and CNCs, as reported by Johar et al. [20]; hence, only the spectrum of CNCs is presented herein.

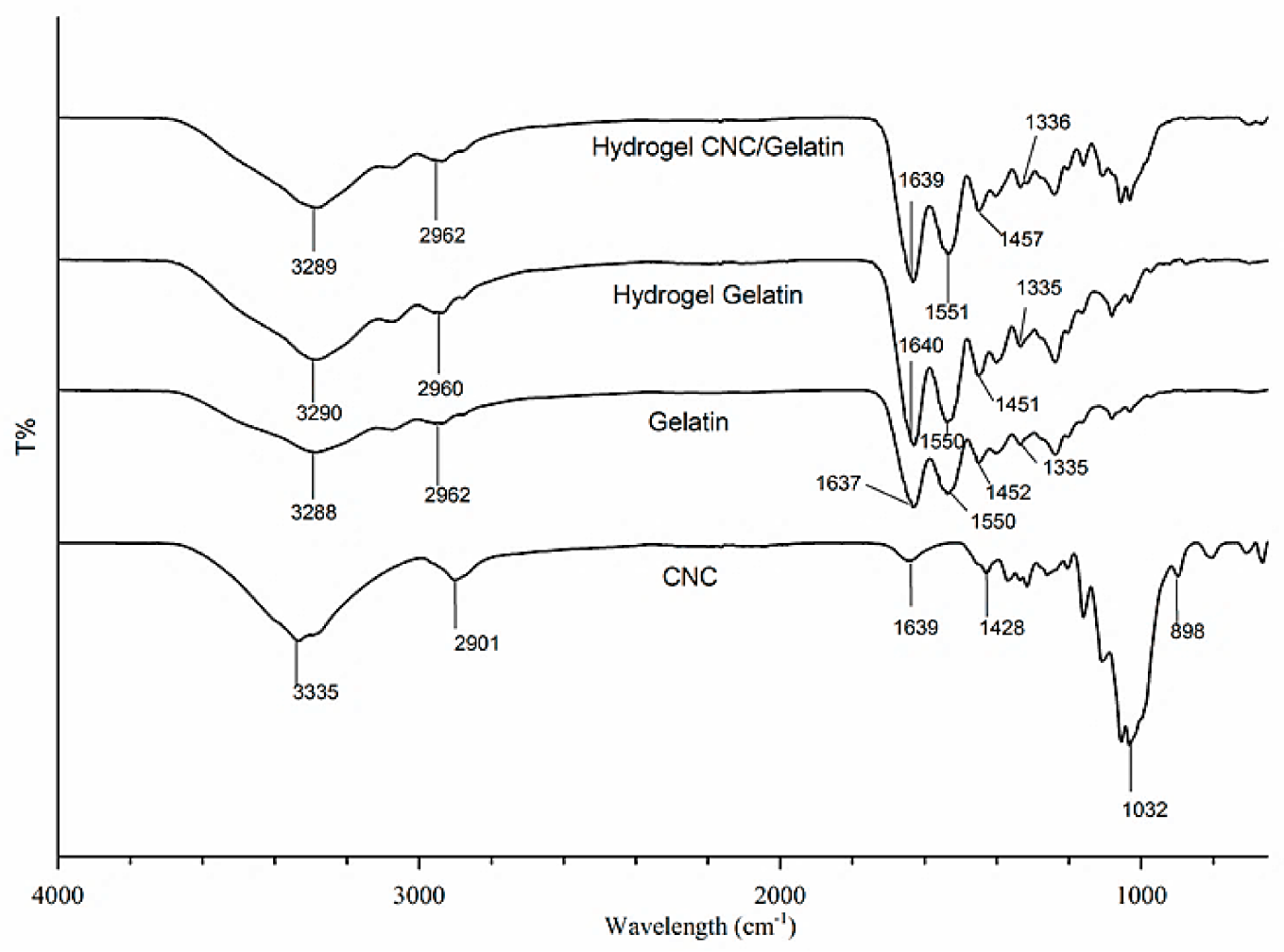

Figure 3. Fourier transform infrared (FTIR) spectra of CNCs, gelatin, and gelatin-based hydrogels.

The FTIR spectrum of gelatin showed absorption peaks at 3288 and $2962 \mathrm{~cm}^{-1}$, which were assigned to $\mathrm{N}-\mathrm{H}$ and aliphatic $\mathrm{C}-\mathrm{H}$ stretches, respectively. Moreover, peaks at 1637 and $1550 \mathrm{~cm}^{-1}$ were ascribed to amide $\mathrm{C}=\mathrm{O}$ stretching (amide I) and amide $\mathrm{N}-\mathrm{H}$ bending (amide II), respectively, in agreement with previous reports [25]. Finally, absorption peaks at around 1452, 1335, and $1240 \mathrm{~cm}^{-1}$ were assigned to $\mathrm{C}-\mathrm{H}$ bending, $\mathrm{C}-\mathrm{N}$ stretching, and $\mathrm{N}-\mathrm{H}$ bending (amide $\mathrm{III}$ ), respectively, as reported by Rokhade et al. [26].

Notably, the spectra of gelatin-based hydrogels featured more intense absorptions than those of pure gelatin, which was attributed to gamma irradiation-induced crosslinking, particularly in the formation of hydrogen bonds between the amino acid residues of polymer chains [3]. Generally, absorption peaks located at $\sim 3290$ and $1550 \mathrm{~cm}^{-1}$ correspond to a triple-helix structure, with their position shift and intensity reduction indicating the breakdown of such structures [8]. On the contrary, crosslinking resulted in an intensity increase of these bands, possibly due to promoting the formation of larger numbers of triple helices. The absorption peaks around $1637 \mathrm{~cm}^{-1}$ also gained intensity as a result of crosslinking, which was ascribed to the increased organization of the helical configuration (as compared to random configuration in the absence of crosslinking). The proposed mechanism for gelatin crosslinking is shown in Figure 4. Gamma-radiation energy is mostly absorbed by water in aqueous solutions; hence, water radiolysis mainly yields reactive species, such as proton radicals $(\mathrm{H})$ and hydroxyl radicals $(\mathrm{OH})$. The most reactive species is conceived by $\mathrm{OH}$, which easily remove $\mathrm{H}$ in 
the polypeptide chain, inducing the formation of gelatin radicals and $\mathrm{H}_{2} \mathrm{O}$ as shown in the propagation step. Gelatin radicals are recombined in the termination step by forming covalent bond between the polypeptide chains. The triple helical structure of gelatin was stabilized by the hydrogen bonds formed between peptide bonds in adjacent chains [5].

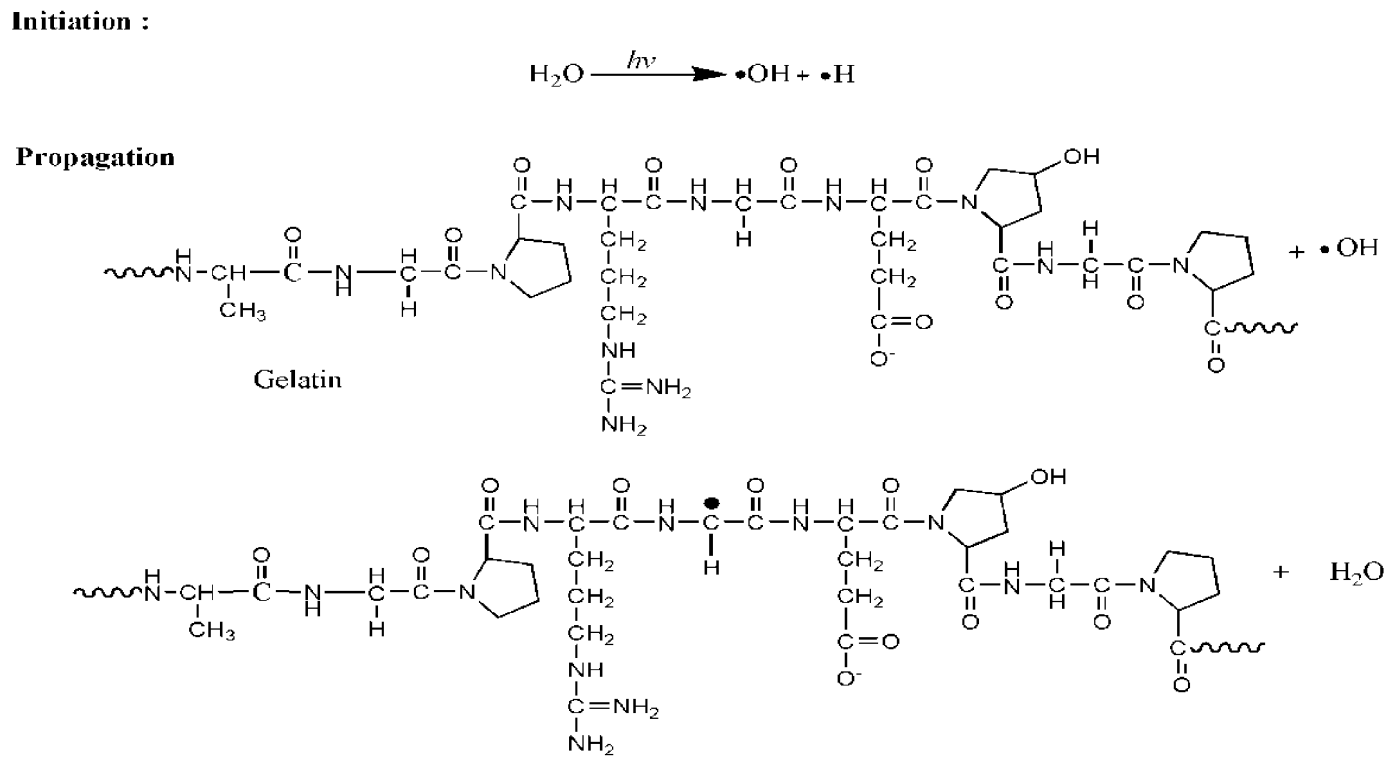

Termination :

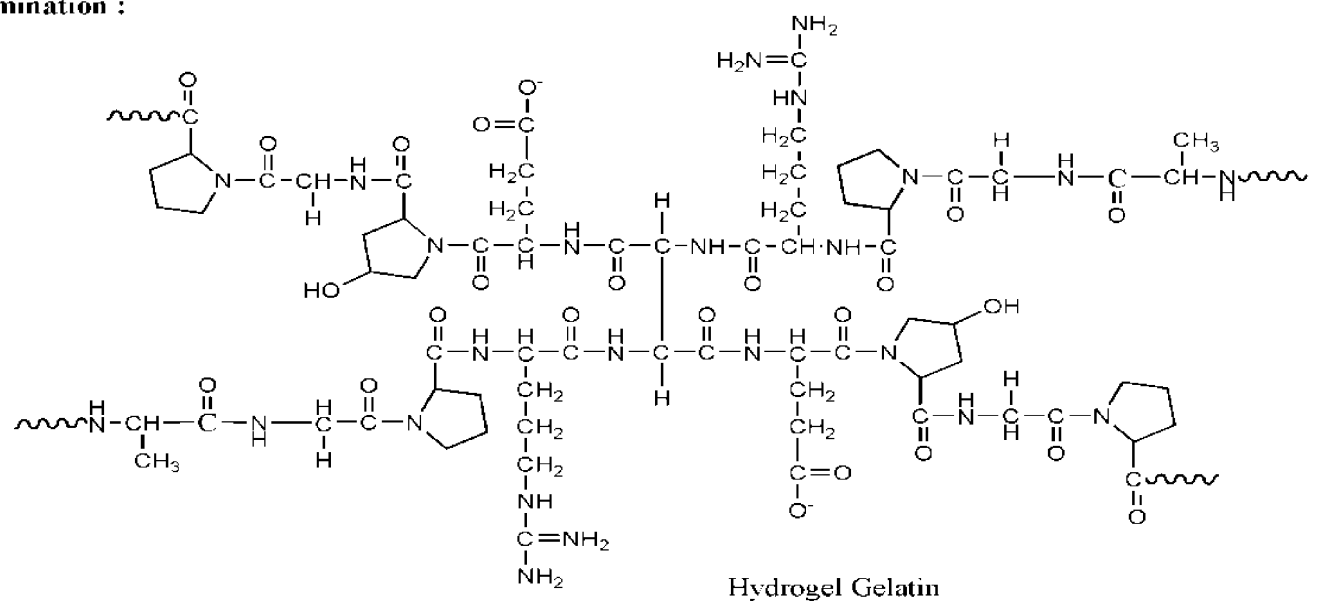

Figure 4. Proposed gelatin-crosslinking mechanism for the formation of hydrogel gelatin by gamma radiation.

The absence of significant differences between the spectra of cellulose- and CNC-containing hydrogels implied that both gelatin and cellulose maintained their individual chemical structures therein, with cellulose/CNCs existing in the form of a semi-IPN (interpenetrating polymer network) [12]. Therefore, the excellent miscibility of cellulose and gelatin allowed the formation of a uniform semi-IPN hydrogel network after gamma irradiation-induced crosslinking [5].

\subsection{Rheological Properties}

The dynamic mechanical properties of hydrogels can be characterized in terms of storage modulus $\left(G^{\prime}\right)$, which is a measure of material elasticity. Figure 5 shows that the frequency-dependent $G^{\prime}$ values of hydrogels A and B generally exceeded those of pure-gelatin hydrogel and unirradiated gelatin, 
which was ascribed to the reinforcing effect of cellulosic fibers. According to Ooi et al. [12], the stiffness of hydrogels increases with an increasing $G^{\prime}$, which makes them more solid-like and, hence, results in better dynamic mechanical properties.

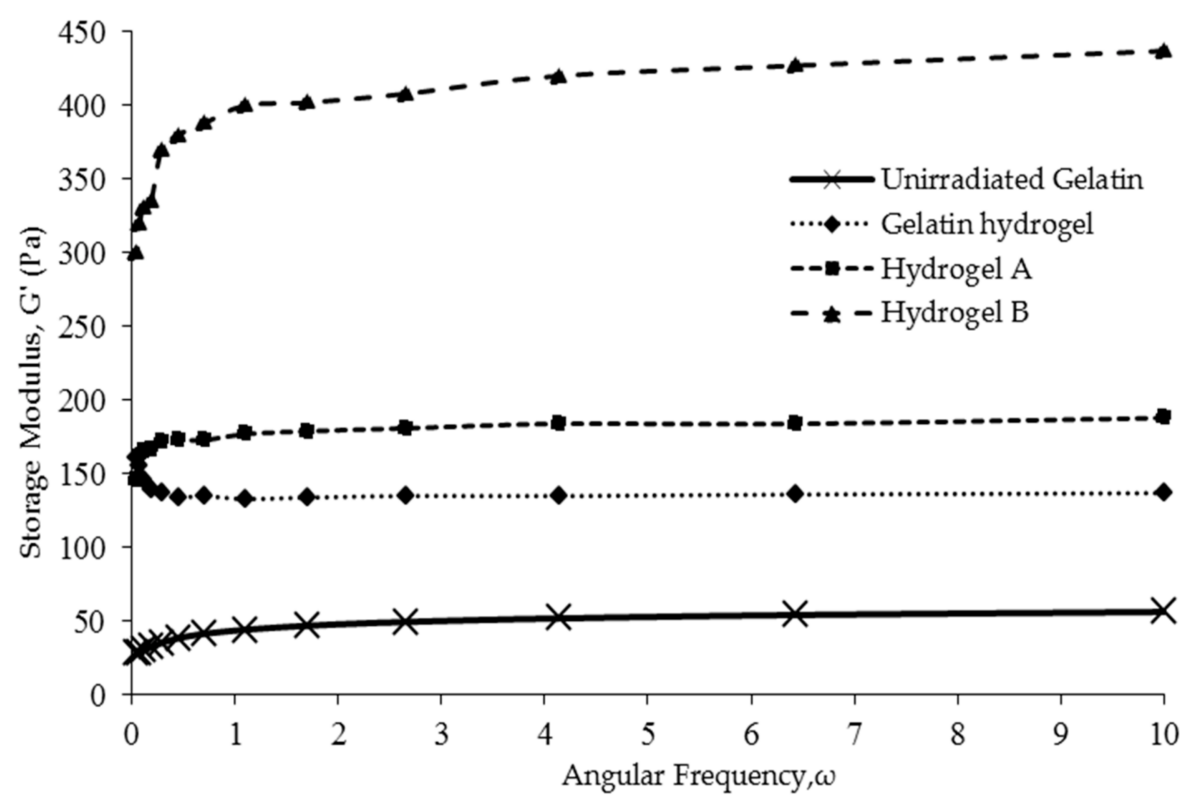

Figure 5. Storage moduli of unirradiated gelatin, gelatin hydrogels, hydrogels A, and hydrogels B.

Figure 5 shows that hydrogels $\mathrm{B}$ had the highest storage modulus compared to hydrogels $\mathrm{A}$ followed by gelatin hydrogels and unirradiated gelatin. Hydrogels B showed a 200\%-250\% higher storage modulus than hydrogel A, which reflected the superiority of the more crystalline CNCs over regular cellulose fibers as reinforcing agents. These results indicate that the higher crystallinity of CNC (Figure 2) had successfully improved hydrogel rigidity and stiffness [12]. Thus, the dynamic mechanical properties of hydrogels might be improved. Similarly, CNCs with a Young's modulus of over $100 \mathrm{GPa}$ and a surface area of several hundred $\mathrm{m}^{2} \mathrm{~g}^{-1}$ have been reported to exhibit impressive mechanical properties and reinforcing capability [11], allowing the preparation of hydrogels perfectly suited for biomedical applications such as drug delivery, wound dressing, and tissue engineering.

However, the incorporation of cellulose microfibrils as reinforcing material in hydrogels B also showed a slight increase on the storage modulus compared to neat hydrogels gelatin. The semicrystalline properties of cellulose itself help as supporting components on the gelatin matrix; hence, a more solid-like gel is formed. As for crosslinking, the gamma-radiation technique surely played its role in inducing crosslinking on gelatin. The storage modulus for unirradiated gelatin is just around $50 \mathrm{~Pa}$ compared to gelatin hydrogel (radiated), which is about $140 \mathrm{~Pa}$. The significant difference of the storage modulus results from the entanglement of the polypeptide chain due to crosslinking [6]. As crosslinking prevailed, the formation of ordered gelatin helical structures increase, hence reducing random coil formation. These ordered configurations hindered the movement of polymer chains, which remarkably improves dynamic mechanical properties and, hence, produces stiffer gelatin hydrogel.

\subsection{Swelling Ratio Test}

Figure 6 shows the swelling behavior of gelatin hydrogels reinforced with cellulose (hydrogel A) and CNCs (hydrogel B), with a filler-free gelatin hydrogel used as a control. During swelling, the diffusion of water molecules into the polymer chain induced the formation of a rubbery (swollen) polymer region by facilitating the relaxation of the polymer network [27]. 
The swelling ratio of all hydrogels increased with time and was lowest for the cellulose-free hydrogel, increasing in the case of cellulose- and CNC-containing hydrogels due to the hydrophilicity of the corresponding fillers. Moreover, cellulosic fibers acted as reinforcing materials and stabilized hydrogel pores during polymer-network relaxation caused by the diffusion of water molecules into hydrogels.

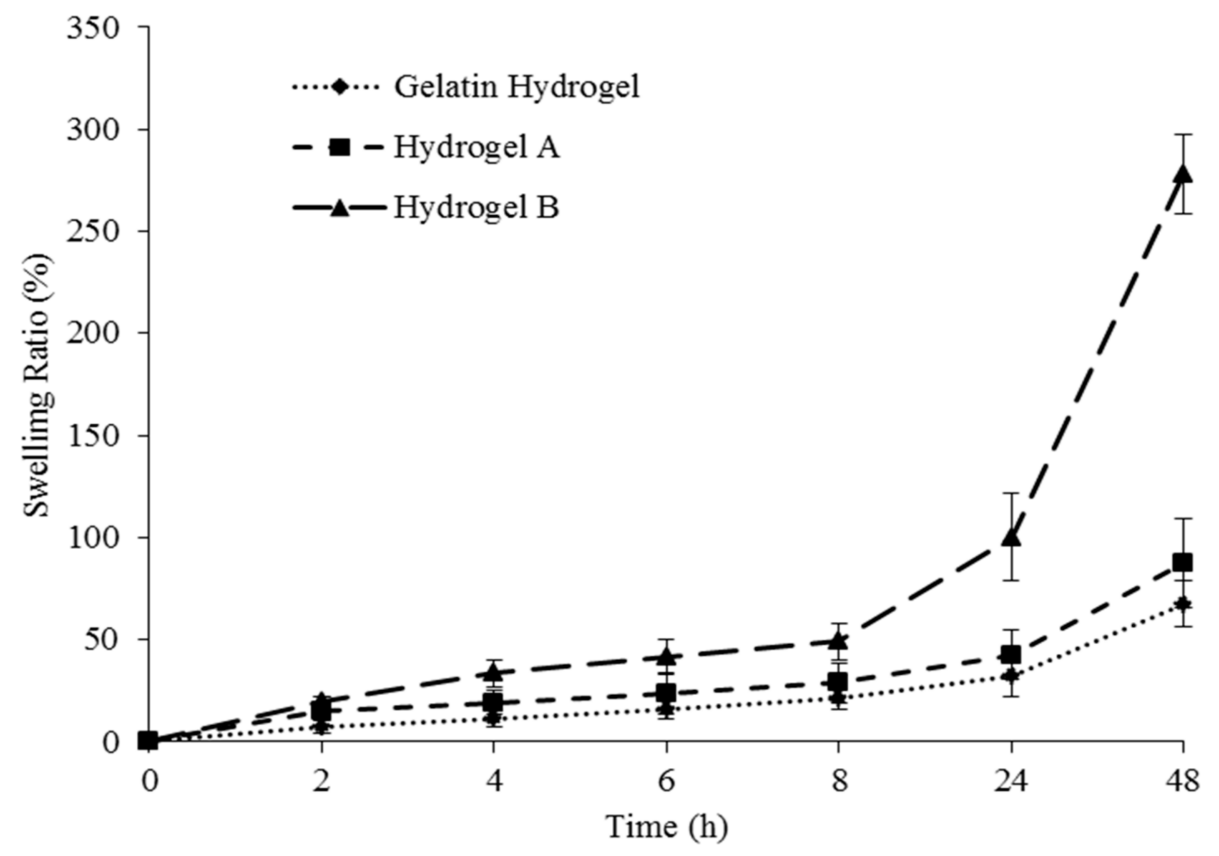

Figure 6. Swelling behavior of different hydrogels.

Hydrogel B achieved a higher swelling ratio than hydrogel A, with the superior water uptake and water-absorption rate of the former ascribed to the smaller and, hence, better-dispersed cellulosic fibers (CNCs) contained therein, facilitating the formation of rigid and stable pores. According to Razmjou et al. [27], the small particle size of CNCs also provides a greater surface area and interstitial volume, allowing the corresponding hydrogels to hold more water and thus resulting in a higher degree of swelling. In addition, the lower swelling degree observed for hydrogel A can be related to the agglomeration of larger cellulose fibers, which weakens hydrogel uniformity.

\subsection{Scanning Electron Microscope (SEM) Analysis}

Figure 7 shows SEM images of pure gelatin, and A and B hydrogels, revealing the morphological differences between swollen and freeze-dried samples and demonstrating that the incorporation of cellulosic fibers generally improved the hydrogel pore structure by affecting pore arrangement, regularity, and rigidity. Thus, cellulosic fibers were concluded to promote the formation of stable pores by supporting the structure of gelatin.

Hydrogel B had more regular and rigidly shaped pores than hydrogel A, which, again, was ascribed to the small size and, hence, better dispersion of CNCs. The thus-produced uniform and structured pore distribution allowed the penetration of water molecules to occur without the destruction of the gel structure-maintaining interactions. Conversely, the irregular pore shape and arrangement observed for hydrogel A was due to the increased dimensions of gelatin gel-filled spaces between cellulose fibers, with the agglomeration of cellulosic fibers resulting in compact pore formation.

Water-absorption speed and water uptake are closely related to the size, structure, and distribution of hydrogel pores. The results of SEM imaging were in good agreement with those obtained by swelling 
degree analysis (Figure 6), being potentially useful for improving the water-absorption capacity/rate of hydrogels [12].

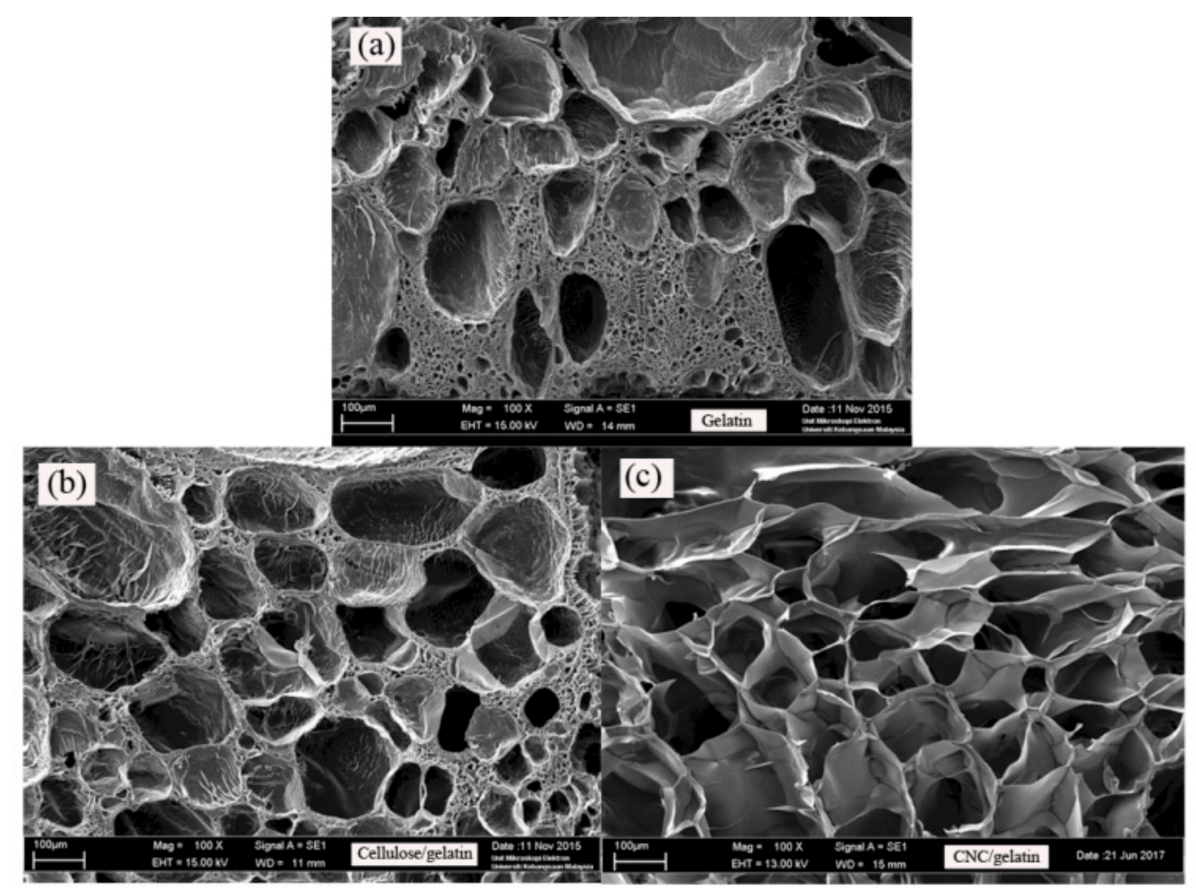

Figure 7. Micrographs of swollen (a) gelatin hydrogel; (b) hydrogel A; and (c) hydrogel B.

\subsection{In Vitro Drug Profiling}

The potential of hydrogels as drug-delivery systems is commonly studied based on in vitro drug loading and release. Herein, only a $\mathrm{CNC} /$ gelatin hydrogel was subjected to the above test due to exhibiting remarkable swelling ability, pore structure, and rheological properties, with the pure gelatin hydrogel used as a control. Figure 8 shows the drug-loading and release efficiencies of gelatin and $\mathrm{CNC}$ /gelatin hydrogel over $24 \mathrm{~h}$, revealing that these efficiencies increased with time in both cases, which indicated that the above hydrogel was potentially suited for use in drug-delivery systems.
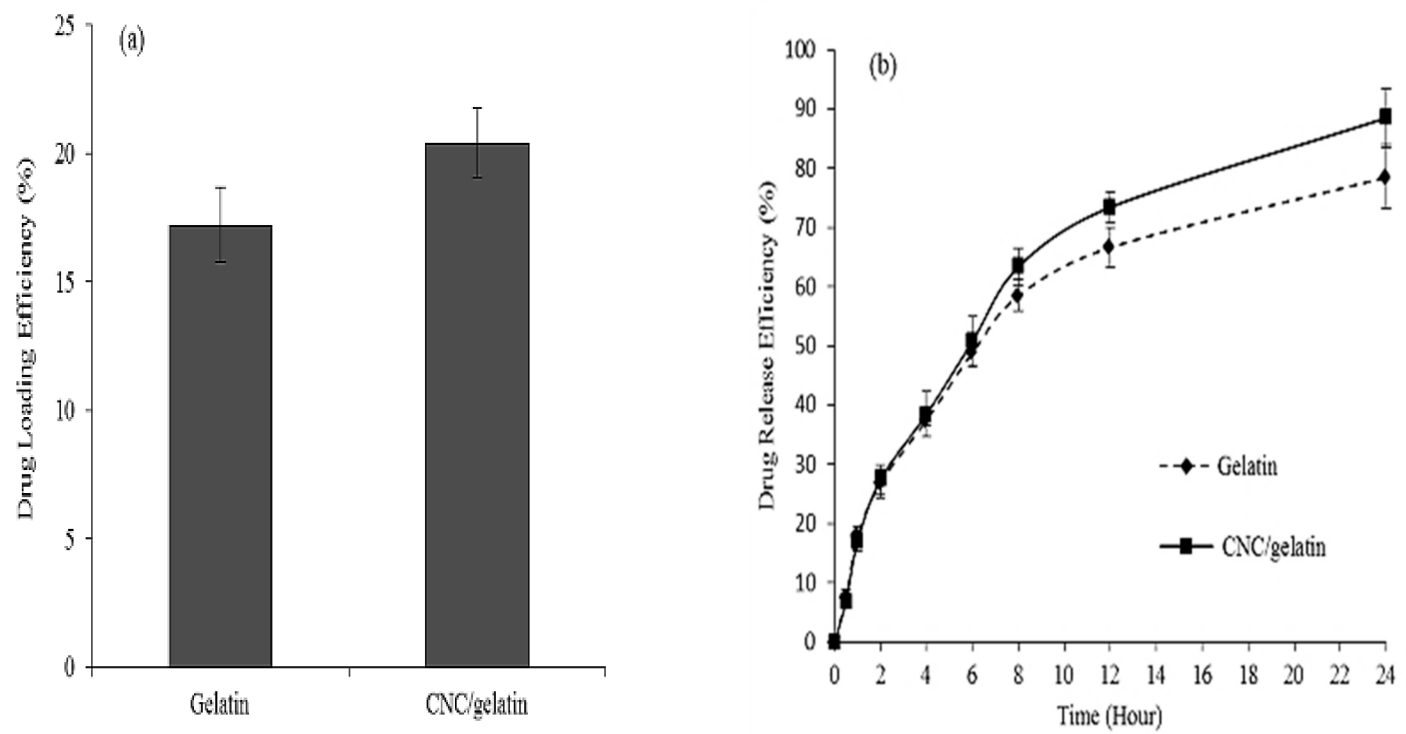

Figure 8. Riboflavin/drug (a) loading and (b) release efficiencies of hydrogels. 
Figure 8 a shows that a riboflavin loading of $20 \%$ was achieved for the $\mathrm{CNC}$ /gelatin hydrogel, whereas a value of only $17 \%$ was observed for the pure-gelatin hydrogel, which indicated the higher drug-loading efficiency of the former. Obviously, the observed trend was coherent with that of the swelling ratio pattern [21]. When hydrogels were immersed in drug solution, penetration of the solvent through their pores induced swelling and thus allowed more drug molecules to be incorporated. Hence, it was concluded that drug-loading efficiency depends on swelling behavior, e.g., the $\mathrm{CNC}$ /gelatin hydrogel was more rigid and exhibited a higher storage modulus and more rigid pores [12] than the gelatin hydrogel, consequently being a better drug carrier due to allowing more drug molecules to diffuse within a given time period.

The efficiency of drug release from the $\mathrm{CNC} /$ gelatin hydrogel exceeded that of the gelatin hydrogel, as shown in Figure 8b, i.e., $73 \%$ and $66 \%$ of riboflavin was released from the above systems within $12 \mathrm{~h}$, respectively, in agreement with the swelling ratio pattern in Figure 6. Herein, drug release was controlled by a solvent-activation or swelling-controlled mechanism, that is, riboflavin trapped within the polymer matrix was released by polymer swelling due to the osmotic effect. Specifically, the osmotic driving force initiated the external water/solvent to enter the drug delivery system and drive out the drug [28].

Based on Figure 7, the increase in swelling ratio was altered by the formation of voids in hydrogels, with the CNC hybridization-promoted formation of regular and rigid voids leading to more efficient drug release. CNCs also acted as strong reinforcing materials controlling the pore structure of gelatin hydrogels, maintaining their rigidity and helping to sustain void formation under the action of the osmotic driving force, thus allowing drug molecules to easily penetrate pores and exit into the buffer solution.

The kinetic mechanism of drug release has been studied by using a mathematical analysis approached on experimental data. An interesting model developed by Peppas-Sahlin [29], which quantifies and materializes the amount of drug released by Fickian diffusion and by polymer relaxation, was chosen. Drug-release data have been treated by Peppas-Sahlin equation:

$$
\frac{M_{\mathrm{t}}}{M_{\infty}}=K_{1} t^{n}+K_{2} t^{2 n}
$$

where $M_{t} / M_{\infty}$ is the fraction of drug release at a time, $t$ and $n$ are diffusional exponents, while $K_{1}$ and $K_{2}$ are kinetic constants. The values of $K_{1}$ indicate the contribution of diffusion (Fickian) and the value of $K_{2}$ is associated with polymer relaxation, respectively. The results of the treated experimental data by the mathematical analysis are recorded in Table 2. The Peppas-Sahlin model indicates that, when the value of exponent $n$ is 0.45 , drug release follows a Fickian-type diffusion mechanism. An abnormal diffusion or non-Fickian diffusion occurs when the value of $n$ is $>0.45$ but smaller than 1 . Meanwhile, when $n=1$, the release kinetic system is known as zero order (transport Case II) [30]. Based on Table 2, the diffusional exponent, $n$ values for this drug kinetic study are between 0.45 and 1 , suggesting an abnormal or non-Fickian diffusion. Therefore, diffusion rate depends on the drug-concentration gradient [21].

Table 2. Fitting paramaters obtained from the Peppas-Sahlin equation.

\begin{tabular}{ccccc}
\hline Hydrogels & Kinetic Constant $\left(\boldsymbol{K}_{\mathbf{1}}\right)$ & Kinetic Constant $\left(\boldsymbol{K}_{\mathbf{2}}\right)$ & Regression Coefficient $\left(\boldsymbol{R}^{\mathbf{2}}\right)$ & $\boldsymbol{n}$ \\
\hline Gelatin & 95.85 & 0.45 & 0.97 & 0.68 \\
CNC/Gelatin & 100.62 & 0.48 & 0.97 & 0.75 \\
\hline
\end{tabular}

The regression coefficient $\left(R^{2}\right)$ is the most common method to assess the fitting of a model equation. It is notable that the $R^{2}$ of the drug-release data is higher than 0.90 , which is high enough to be evaluated with the Peppas-Sahlin model. The value of diffusion constant $K_{1}$ is remarkably higher than the value of relaxation constant $K_{2}$ for both hydrogels, which indicates the predominance of the swelling mechanism over the erosion mechanism combined with the high solubility of drugs [21]. 


\section{Conclusions}

Herein, we describe the preparation of crosslinked gelatin/cellulose-containing hydrogels, utilizing gamma irradiation for chemical reagent-free crosslinking and employing cellulose fibers isolated from rice husks and CNCs as hydrogel-reinforcing agents. Compared to that of regular fibrous cellulose, incorporation of CNCs resulted in higher swelling ability, better dynamic mechanical properties, and enhanced drug uptake/release performance, i.e., the size of included cellulose markedly affected the performance of crosslinked hydrogels, in agreement with our original hypothesis. $\mathrm{CNC} /$ gelatin hydrogels produced are potentially suitable for drug-delivery applications, suggesting a future extended direction of the present method.

Author Contributions: W.H.W.I. performed the experiment, analyzed the data, and wrote the paper. The work was supervised by I.A. and cosupervised by S.R. and M.C.I.M.A. The draft of the manuscript was reviewed and revised by I.A., S.R., and M.C.I.M.A. The manuscript was submitted by I.A.

Acknowledgments: The authors acknowledge financial support from Universiti Kebangsaan Malaysia and the Ministry of Higher Education (MOHE) under FRGS-MRSA/1/2016/STG07/UKM/01/1 and DIP-2016-026.

Conflicts of Interest: The authors declare no conflict of interest.

\section{References}

1. Varghese, J.S.; Chellappa, N.; Fathima, N.N. Gelatin-carrageenan hydrogels: Role of pore size distribution on drug delivery process. Colloids Surf. B Biointerfaces 2014, 113, 346-351. [CrossRef] [PubMed]

2. Haema, K.; Oyama, T.G.; Kimura, A.; Taguchi, M. Radiation stability and modification of gelatin for biological and medical applications. Radiat. Phys. Chem. 2014, 103, 126-130. [CrossRef]

3. Islam, M.M.; Zaman, A.; Islam, M.S.; Khan, M.A.; Rahman, M.M. Physico-chemical characteristics of gamma-irradiated gelatin. Prog. Biomater. 2014, 3, 21. [CrossRef] [PubMed]

4. Gulrez, S.K.; Al-Assaf, S.; Phillips, G.O. Hydrogels: Methods of preparation, characterisation and applications. In Progress in Molecular and Environmental Bioengineering from Analysis and Modeling to Technology Applications; InTech: London, UK, 2011.

5. Yang, C.; Xu, L.; Zhou, Y.; Zhang, X.; Huang, X.; Wang, M.; Han, Y.; Zhai, M.; Wei, S.; Li, J. A green fabrication approach of gelatin/CM-chitosan hybrid hydrogel for wound healing. Carbohydr. Polym. 2010, 82, 1297-1305. [CrossRef]

6. Zhang, X.; Xu, L.; Huang, X.; Wei, S.; Zhai, M. Structural study and preliminary biological evaluation on the collagen hydrogel crosslinked by $\gamma$-irradiation. J. Biomed. Mater. Res. Part A 2012, 100, 2960-2969. [CrossRef] [PubMed]

7. Eid, M.; Abdel-Ghaffar, M.A.; Dessouki, A.M. Effect of maleic acid content on the thermal stability, swelling behaviour and network structure of gelatin-based hydrogels prepared by gamma irradiation. Nucl. Instrum. Methods Phys. Res. Sect. B Beam Interact. Mater. Atoms 2009, 267, 91-98. [CrossRef]

8. Wisotzki, E.I.; Hennes, M.; Schuldt, C.; Engert, F.; Knolle, W.; Decker, U.; Käs, J.A.; Zink, M.; Mayr, S.G. Tailoring the material properties of gelatin hydrogels by high energy electron irradiation. J. Mater. Chem.B 2014, 2, 4297-4309. [CrossRef]

9. Arthur, J.C., Jr.; Hinojosa, O.; Tripp, V.W. Effect of crystalline structure on the trapped radical spectra of irradiated cellulose. J. Appl. Polym. Sci. 1969, 13, 1497-1507. [CrossRef]

10. Lepore, A.; Baccaro, S.; Casieri, C.; Cemmi, A.; De Luca, F. Role of water in the ageing mechanism of paper. Chem. Phys. Lett. 2012, 531, 206-209. [CrossRef]

11. Dufresne, A. Nanocellulose: A new ageless bionanomaterial. Mater. Today 2013, 16, 220-227. [CrossRef]

12. Ooi, S.Y.; Ahmad, I.; Amin, M.C.I.M. Cellulose nanocrystals extracted from rice husks as a reinforcing material in gelatin hydrogels for use in controlled drug delivery systems. Ind. Crops Prod. 2016, 93, 227-234. [CrossRef]

13. Ajji, Z.; Othman, I.; Rosiak, J.M. Production of hydrogel wound dressings using gamma radiation. Nucl. Instrum. Methods Phys. Res. Sect. B Beam Interact. Mater. Atoms 2005, 229, 375-380. [CrossRef]

14. Kuen, C.Y.; Fakurazi, S.; Othman, S.S.; Masarudin, M.J. Increased Loading, Efficacy and Sustained Release of Silibinin, a Poorly Soluble Drug Using Hydrophobically-Modified Chitosan Nanoparticles for Enhanced Delivery of Anticancer Drug Delivery Systems. Nanomaterials 2017, 7, 379. [CrossRef] [PubMed] 
15. El-Sherbiny, I.M.; Yacoub, M.H. Hydrogel scaffolds for tissue engineering: Progress and challenges. Glob. Cardiol. Sci. Pract. 2013, 2013, 316-342. [CrossRef] [PubMed]

16. Kargarzadeh, H.; Johar, N.; Ahmad, I. Starch biocomposite film reinforced by multiscale rice husk fiber. Compos. Sci. Technol. 2017, 151, 147-155. [CrossRef]

17. Food, Administration, D. CFR-Code of Federal Regulations, Title 21. US Department of Health and Human Services, 2013. Available online: https:/ / www.accessdata.fda.gov/scripts/cdrh/cfdocs/cfcfr/CFRSearch. Cfm (accessed on 19 September 2018).

18. Feil, H.; Bae, Y.H.; Feijen, J.; Kim, S.W. Molecular separation by thermosensitive hydrogel membranes. J. Membr. Sci. 1991, 64, 283-294. [CrossRef]

19. Smith, D.J. Characterization of Nanomaterials Using Transmission Electron Microscopy. Nanocharacterisation 2015. [CrossRef]

20. Johar, N.; Ahmad, I.; Dufresne, A. Extraction, preparation and characterization of cellulose fibres and nanocrystals from rice husk. Ind. Crops Prod. 2012, 37, 93-99. [CrossRef]

21. Lim, L.S.; Rosli, N.A.; Ahmad, I.; Mat Lazim, A.; Mohd Amin, M.C.I. Synthesis and swelling behavior of $\mathrm{pH}$-sensitive semi-IPN superabsorbent hydrogels based on poly (acrylic acid) reinforced with cellulose nanocrystals. Nanomaterials 2017, 7, 399. [CrossRef] [PubMed]

22. Du, L.; Wang, J.; Zhang, Y.; Qi, C.; Wolcott, M.P.; Yu, Z. Preparation and characterization of cellulose nanocrystals from the bio-ethanol residuals. Nanomaterials 2017, 7, 51. [CrossRef] [PubMed]

23. Sheltami, R.M.; Abdullah, I.; Ahmad, I.; Dufresne, A.; Kargarzadeh, H. Extraction of cellulose nanocrystals from mengkuang leaves (Pandanus tectorius). Carbohydr. Polym. 2012, 88, 772-779. [CrossRef]

24. Alemdar, A.; Sain, M. Isolation and characterization of nanofibers from agricultural residues-Wheat straw and soy hulls. Bioresour. Technol. 2008, 99, 1664-1671. [CrossRef] [PubMed]

25. Aewsiri, T.; Benjakul, S.; Visessanguan, W. Functional properties of gelatin from cuttlefish (Sepia pharaonis) skin as affected by bleaching using hydrogen peroxide. Food Chem. 2009, 115, 243-249. [CrossRef]

26. Rokhade, A.P.; Agnihotri, S.A.; Patil, S.A.; Mallikarjuna, N.N.; Kulkarni, P.V.; Aminabhavi, T.M. Semi-interpenetrating polymer network microspheres of gelatin and sodium carboxymethyl cellulose for controlled release of ketorolac tromethamine. Carbohydr. Polym. 2006, 65, 243-252. [CrossRef]

27. Razmjou, A.; Simon, G.P.; Wang, H. Effect of particle size on the performance of forward osmosis desalination by stimuli-responsive polymer hydrogels as a draw agent. Chem. Eng. J. 2013, 215-216, 913-920. [CrossRef]

28. Langer, R.; Peppas, N.A. Advances in biomaterials, drug delivery, and bionanotechnology. AIChE J. 2003, 49, 2990-3006. [CrossRef]

29. Peppas, N.A.; Sahlin, J.J. A simple equation for the description of solute release. III. Coupling of diffusion and relaxation. Int. J. Pharm. 1989, 57, 169-172. [CrossRef]

30. Mady, O. Mechanisms and percent of drug release of each new mathematic approach. Int. Res. J. Pharm. Appl. Sci. 2013, 3, 56-69.

(C) 2018 by the authors. Licensee MDPI, Basel, Switzerland. This article is an open access article distributed under the terms and conditions of the Creative Commons Attribution (CC BY) license (http:/ / creativecommons.org/licenses/by/4.0/). 